PROCEEDINGS OF THE

AMERICAN MATHEMATICAL SOCIETY

Volume 129, Number 2, Pages 539-542

S 0002-9939(00)05592-1

Article electronically published on September 20, 2000

\title{
THE RIESZ DECOMPOSITION PROPERTY FOR THE SPACE OF REGULAR OPERATORS
}

\author{
NICOLAE DĂNEŢ \\ (Communicated by David R. Larson) \\ Dedicated to Prof. Romulus Cristescu on his 70th birthday
}

\begin{abstract}
If $E$ and $F$ are Banach lattices such that $E$ is separable and $F$ has the countable interpolation property, then the space of all continuous regular operators $\mathcal{L}^{r}(E, F)$ has the Riesz decomposition property. This result is a positive answer to a conjecture posed by A. W. Wickstead.
\end{abstract}

\section{INTRODUCTION}

It is well known that the space of all regular operators $L^{r}(E, F)$ between two Riesz spaces $E$ and $F$ is a Riesz space if the range space $F$ is Dedekind complete. (Actually, the space $L^{r}(E, F)$ is a Dedekind complete Riesz space (4, Th.1.3.2).) If $F$ is not a Dedekind complete Riesz space, there exists little knowledge about the order structure of the space $L^{r}(E, F)$. In [1] Y. A. Abramovich and A. W. Wickstead showed that the space $L^{r}\left(l_{0}^{\infty}, l_{0}^{\infty}\right)$ fails to have the Riesz decomposition property. $\left(l_{0}^{\infty}\right.$ is the space of all real sequences which are constant except for a finite number of terms and it is the smallest possible non-Dedekind complete Riesz space.) In [6] A. W. Wickstead began the study of the Riesz decomposition property in the space of continuous regular operators between Banach lattices. He proved that the space $\mathcal{L}^{r}(c, F)$ has the Riesz decomposition property if and only if the Banach lattice $F$ has the countable interpolation property. He showed also that it is possible for such a space to have the Riesz decomposition property without being a lattice, and that not all spaces of regular operators have the Riesz decomposition property.

In this paper we give a positive answer to the following conjecture posed by A. W. Wickstead in [6]: the space of all continuous regular operators $\mathcal{L}^{r}(E, F)$ between the Banach lattices $E$ and $F$ has the Riesz decomposition property whenever $E$ is separable and $F$ has the countable interpolation property.

For the unexplained terms in the theory of Banach lattices and positive operators we refer to [4].

\section{Some DEFINITIONS AND A PRELIMINARY RESUlT}

We say that the ordered vector space $E$ has the Riesz decomposition property if there exist $x_{1}, x_{2} \in E$ such that $0 \leq x_{1} \leq z_{1}, 0 \leq x_{2} \leq z_{2}$ and $x=x_{1}+x_{2}$ for

Received by the editors December 17, 1998 and, in revised form, May 4, 1999.

1991 Mathematics Subject Classification. Primary 47B60; Secondary 47B65, 46B42, 47L05.

Key words and phrases. Banach lattices, regular operators, Riesz decomposition property.

(C)2000 American Mathematical Society 
$x, z_{1}, z_{2} \in E_{+}$and $0 \leq x \leq z_{1}+z_{2}$. Every Riesz space $E$ has the Riesz decomposition property.

We recall that an Archimedean Riesz space $F$ has the countable interpolation property (or Cantor property) if for all sequences $\left(x_{n}\right)$ and $\left(z_{n}\right)$ in $F$ such that $x_{n} \leq z_{m}$ for all $n, m \in \mathbb{N}$ there exists $y \in F$ such that $x_{n} \leq y \leq z_{m}$ for all $n, m \in \mathbb{N}$ (equivalently, if, whenever $x_{n} \uparrow, z_{n} \downarrow$ and $x_{n} \leq z_{m}$ for all $n, m \in \mathbb{N}$, there exists $y \in F$ such that $x_{n} \leq y \leq z_{n}$ for all $n \in \mathbb{N}$ ). C. B. Huijsmans and B. de Pagter have shown that an Archimedean Riesz space $F$ has the countable interpolation property if and only if $F$ is uniformly complete and $F=\left\{x^{+}\right\}^{\perp}+\left\{x^{-}\right\}^{\perp}$ for all $x \in F$ ([3], Th. 9.15). If $X$ is a completely regular Hausdorff space, then the space $C(X)$ has countable interpolation property if and only if $X$ is an F-space, i.e., any pair of disjoint open $F_{\sigma}$ subsets of $X$ have disjoint closures $([5)$. For other equivalent conditions with the countable interpolation property for $C(X)$ see 3, Th. 10.5.

Using the countable interpolation property, Y. A. Abramovich and A. W. Wickstead proved in 2 ] the following new version of the Hahn-Banach-Kantorovich extension theorem.

Theorem 2.1 ([2], Th. 3.5). Let $E$ and $F$ be Banach lattices, such that $E$ is separable and $F$ has the countable interpolation property, and let $P: E \rightarrow F$ be a continuous sublinear operator. If $G$ is a vector subspace of $E$ and $T: G \rightarrow F$ is a continuous linear operator satisfying $T(x) \leq P(x)$ for all $x \in G$, then there exists an extension $\hat{T}$ of $T$ to all of $E$ also satisfying $\hat{T}(x) \leq P(x)$ for all $x \in E$.

We mention that by a sublinear operator we understand an operator $P: E \rightarrow F$ that is subadditive and positively homogeneous, i.e., $P\left(x_{1}+x_{2}\right) \leq P\left(x_{1}\right)+P\left(x_{2}\right)$ and $P(\alpha x)=\alpha P(x)$ for all $x_{1}, x_{2}, x \in E$ and $\alpha \geq 0$. In 2] the authors use the name of sublinear operator for an operator that is subadditive and $P(\alpha x)=|\alpha| P(x)$ for all $x \in E$ and $\alpha \in \mathbb{R}$. But their proof works for a sublinear operator too.

Let $E$ and $F$ be two Archimedean Riesz spaces. A linear operator $T: E \rightarrow F$ is called positive if $T(x) \geq 0$ for all $x \in E_{+}$. We will denote this by $T \geq 0$. $T$ is called regular if $T$ is the difference of two positive linear operators. We denote by $L^{r}(E, F)$ the space of all regular operators. If $E$ is a Banach lattice and $F$ a normed Riesz space, then every positive linear operator is continuous (4, Proposition 1.3.5). In this situation we have $L^{r}(E, F)=\mathcal{L}^{r}(E, F)$, where $\mathcal{L}^{r}(E, F)$ denotes the space of all continuous regular operators.

\section{THE MAIN THEOREM}

The goal of this section is to demonstrate the following theorem.

Theorem 3.1. Let $E$ and $F$ be two Banach lattices such that $E$ is separable and $F$ has the countable interpolation property. Then the space of all continuous regular operators $\mathcal{L}^{r}(E, F)$ has the Riesz decomposition property.

Proof. The proof follows the idea of demonstration of Corollary 1.5.6 from [4] and uses the extension Theorem 2.1. It is quite different from Wickstead's proof for the particular case $E=c$.

Let $T, S_{1}, S_{2}$ be three positive linear operators in $\mathcal{L}^{r}(E, F)$ such that

$$
T \leq S_{1}+S_{2} .
$$


We have to prove that there exist two linear operators $T_{1}, T_{2} \in \mathcal{L}^{r}(E, F)$ with the properties

$$
\begin{aligned}
0 & \leq T_{i} \leq S_{i}, \quad i=1,2, \\
T & =T_{1}+T_{2} .
\end{aligned}
$$

Consider the Banach lattice $E \times E$ with the canonical order and norm. Define $P: E \times E \rightarrow F$ by

$$
P\left(x_{1}, x_{2}\right)=S_{1}\left(x_{1}^{+}\right)+S_{2}\left(x_{2}^{+}\right), \quad\left(x_{1}, x_{2}\right) \in E \times E .
$$

$P$ is a sublinear operator. Since the lattice operations are continuous and $S_{1}, S_{2}$ are continuous operators, it follows that $P$ is continuous too.

Consider now the subspace $G=\{(x, x) \mid x \in E\}$ of $E \times E$ and define on $G$ the operator $T_{0}: G \rightarrow F$ by

$$
T_{0}(x, x)=T(x), \quad x \in E .
$$

$T_{0}$ is a continuous linear operator and

$$
\begin{aligned}
T_{0}(x, x) & =T(x)=T\left(x^{+}\right)-T\left(x^{-}\right) \leq T\left(x^{+}\right) \\
& \leq S_{1}\left(x^{+}\right)+S_{2}\left(x^{+}\right)=P(x, x)
\end{aligned}
$$

on $G$. By Theorem 2.1 there exists an extension $\hat{T}$ of $T_{0}$ to all $E \times E$ which satisfies the inequality

$$
\hat{T}\left(x_{1}, x_{2}\right) \leq P\left(x_{1}, x_{2}\right), \quad\left(x_{1}, x_{2}\right) \in E \times E .
$$

Define

$$
\begin{array}{ll}
T_{1}(x)=\hat{T}(x, 0), & T_{1}: E \rightarrow F, \\
T_{2}(x)=\hat{T}(0, x), & T_{2}: E \rightarrow F .
\end{array}
$$

$T_{1}, T_{2}$ are the desired operators. Indeed, for every $x \in E$ we have

$$
\begin{aligned}
T_{1}(x) & =\hat{T}(x, 0) \leq P(x, 0)=S_{1}\left(x^{+}\right), \\
-T_{1}(x) & =T_{1}(-x)=\hat{T}(-x, 0) \leq P(-x, 0)=S_{1}\left((-x)^{+}\right)=S_{1}\left(x^{-}\right),
\end{aligned}
$$

from which it follows that

$$
-S_{1}\left(x^{-}\right) \leq T_{1}(x) \leq S_{1}\left(x^{+}\right), \quad x \in E .
$$

If $x \geq 0$, then $x^{+}=x$ and $x^{-}=0$. Therefore, we obtain

$$
0 \leq T_{1}(x) \leq S_{1}(x), \quad x \in E_{+} .
$$

Similarly $0 \leq S_{2} \leq T_{2}$. Finally, we have

$$
T(x)=T_{0}(x, x)=\hat{T}(x, x)=\hat{T}(x, 0)+\hat{T}(0, x)=T_{1}(x)+T_{2}(x)
$$

for all $x \in E$. The proof is complete.

Finally, we can give the following characterization of a Banach lattice $F$ which has the countable interpolation property. The implication (iii) $\Rightarrow$ (i) is proved in [6], Theorem 3.1.

Corollary 3.2. The following conditions on a Banach lattice $F$ are equivalent:

(i) $F$ has the countable interpolation property;

(ii) For every separable Banach lattice $E$ the space $\mathcal{L}^{r}(E, F)$ has the Riesz decomposition property;

(iii) $\mathcal{L}^{r}(c, F)$ has the Riesz decomposition property. 


\section{REFERENCES}

[1] Y. A. Abramovich and A. W. Wickstead, Regular operators from and into a small Riesz space, Indag. Mathem., N.S. 2 (1991), no.3, 257-274. MR 93i:47052

[2] Y. A. Abramovich and A. W. Wickstead, The regularity of order bounded operators into $C(K)$, II, Quart. J. Math. Oxford (2), 44 (1993), 257-270. MR 94h:47067

[3] C. B. Huijsmans and B. de Pagter, On z-ideals and d-ideals in Riesz spaces, II, Indag. Mathem. 42 (1980), no.4, 391-408. MR 83c:46004a

[4] Peter Meyer-Nieberg, Banach Lattices, Springer-Verlag, Berlin Heidelberg New York, 1991. MR 93f:46025

[5] G. L. Seever, Measures on F-spaces, Trans. Amer. Math. Soc.133 (1968), 267-280. MR 37:1976

[6] A. W. Wickstead, Spaces of operators with the Riesz separation property, Indag. Mathem., N.S. 6 (1995), no.2, 235-245. MR 96g:47032

Technical University of Civil Engineering of Bucharest, 122-124, Lacul Tei Blvd., 72302 Bucharest, Romania

E-mail address: ndanet@fx.ro 